
Sustainable Cities Research Group

\title{
The Impact of Trees on Building Energy Use
}

Ulrike Passe, Janette Thompson, Baskar Ganapathysubramanian

Post-docs and Students: Boshun Gao, Breanna Marmur, Saha Homagni, Himanshu Sharma

\section{Background}

Extreme heat events are predicted to increase in frequency and duration in the Midwest region of the United States by the year 2050 (Melillo 2014). Trees in urban areas can help mitigate heat by reducing solar radiation loads (due to canopy size, shape, leaf area, and leaf density), as well as through careful tree placement (Fig. 1) (Hwang 2017). Additionally, evapotranspiration (ET) from tree leaves can help to cool the microclimate around the trees, thereby reducing heat loads and building energy use (Nowak 2017).

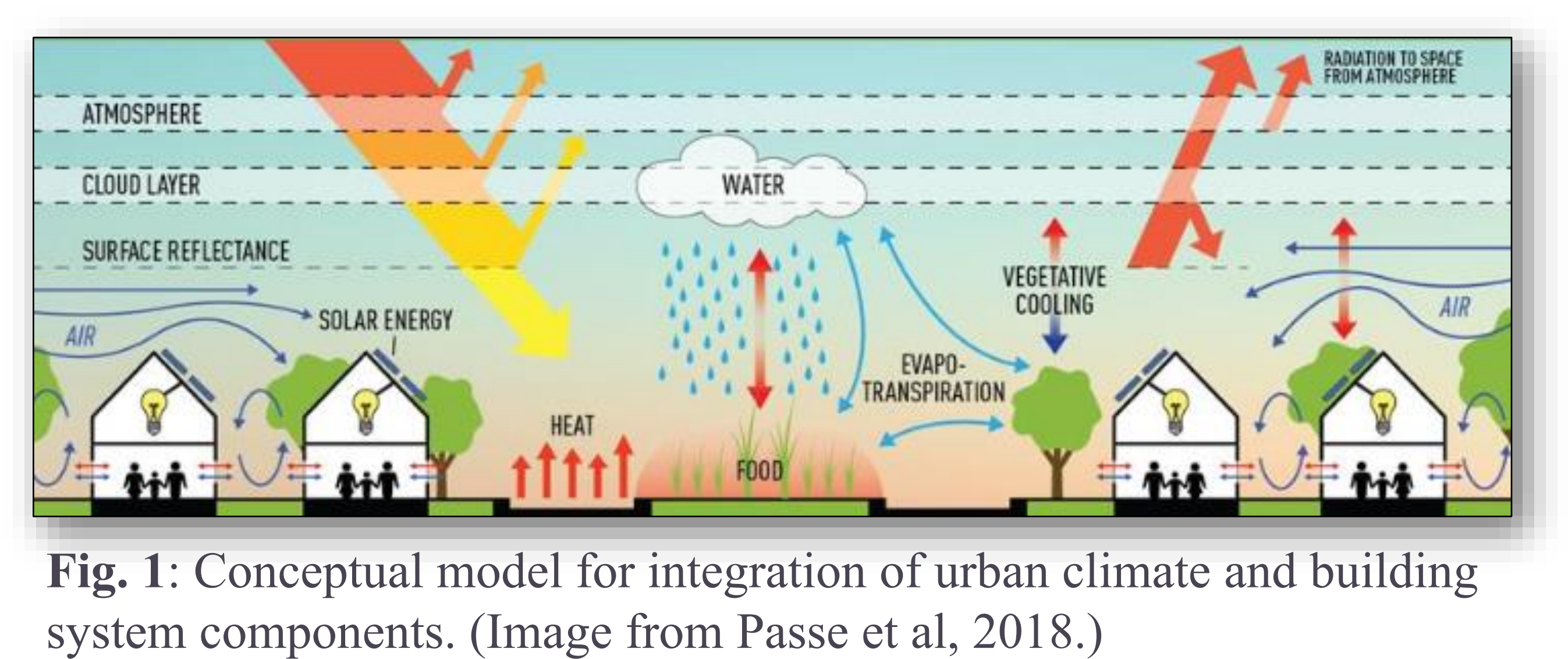

Fig. 1: Conceptual model for integration of urban climate and building

\section{Research Goals}

Collect empirical data on local trees and buildings in a Des Moines, IA neighborhood (Capitol East)

- Estimate leaf temperatures and evapotranspiration rates based on a literature search and climatic conditions in Des Moines

- Conduct shadow range analysis by incorporating empirical data in an urban modeling interface (umi) (Reinhart 2018).

- Estimate the effects of trees on air flow and building temperatures using computational fluid dynamics (CFD) models

\section{Tree and Building Inventory}

A complete inventory of 1,142 trees and 340 buildings was conducted in the Capitol East Neighborhood of Des Moines, Iowa (estimated 25.2\% canopy cover, Fig. 2).

- Tree data included species, diameter, height, canopy shape, and percent canopy filled

- Assembled parcel and building data from the City of Des Moines Assessor's Office records

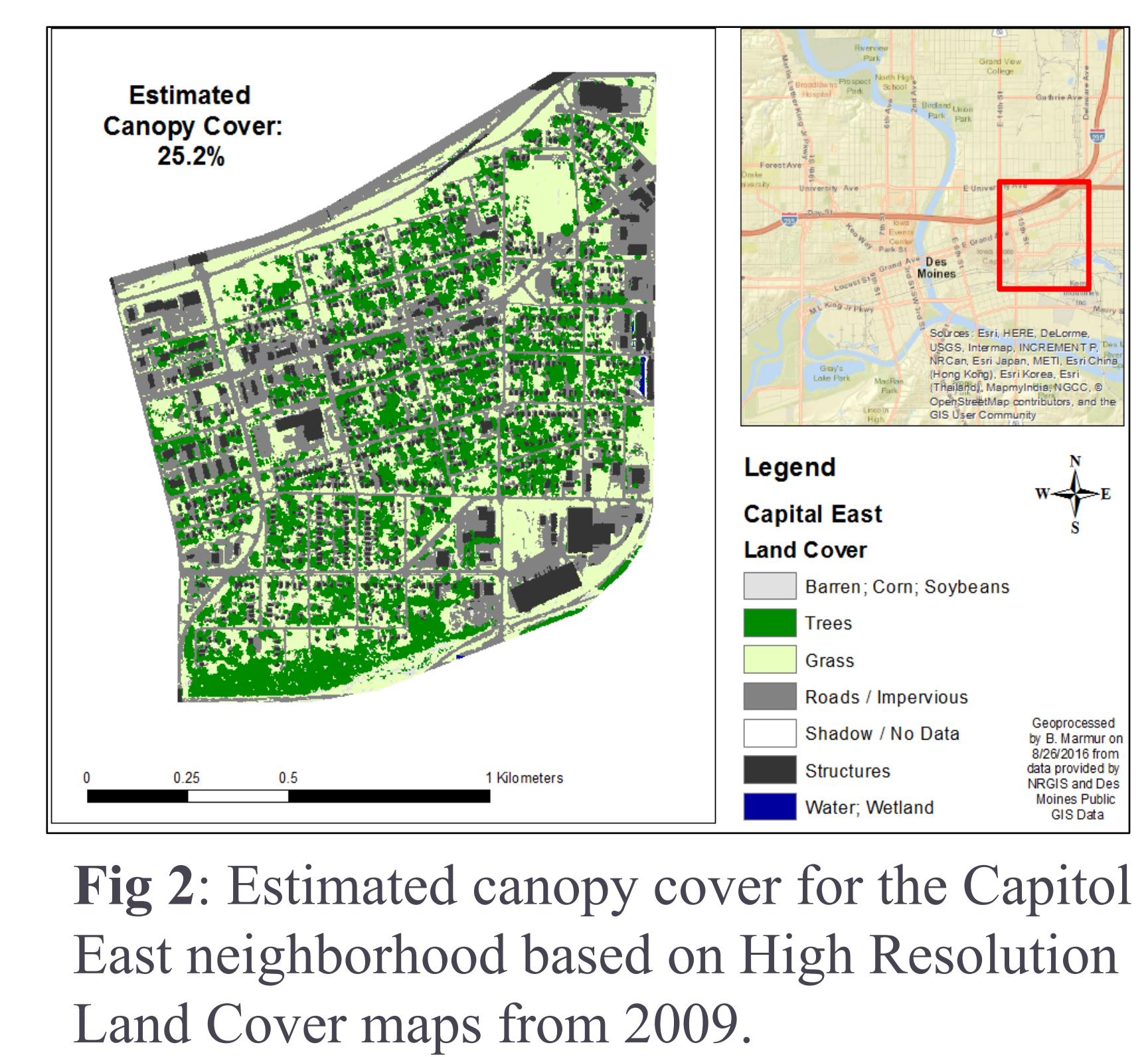

Evapotranspiration and Leaf Temperature Estimation

ET can cool the area near trees. Tree ET was estimated (Eq. 1) using the FAO Penman-Monteith equation for proxy species selected from the literature for Kc (crop coefficient), and standard FAO ET Calculator values for $\mathrm{ET}_{\text {ref }}$ (Allen $1998 ; \mathrm{FAO}-\mathrm{UN}$ n.d.).

- ET approximations were made for representative small (crapapple), medium (ornamental pear), and large (American basswood) trees in the spring, summer, and fall (Table 1).

$$
E T=K_{c} E T_{r e f}
$$

Eq. 1: Empirically derived equation for
estimating species evaporation based on the FAO Penmen-Monteith equation reference ET $\left(\mathrm{ET}_{\text {ref } \_}\right)$and a crop coefficient $\left(\mathrm{K}_{\mathrm{C}}\right)$.

\section{GIS and umi Integration}

Shadow range analysis was used to estimate hours of direct sunlight received by buildings:

- GIS data were incorporated in Meerkat (a GIS data plug-in) and integrated in umi to generate a visualization model (Fig. 4a)

- The Rhinoceros model provided input to umi for simulation of building energy performance using shadow range analysis (Fig. 4b)
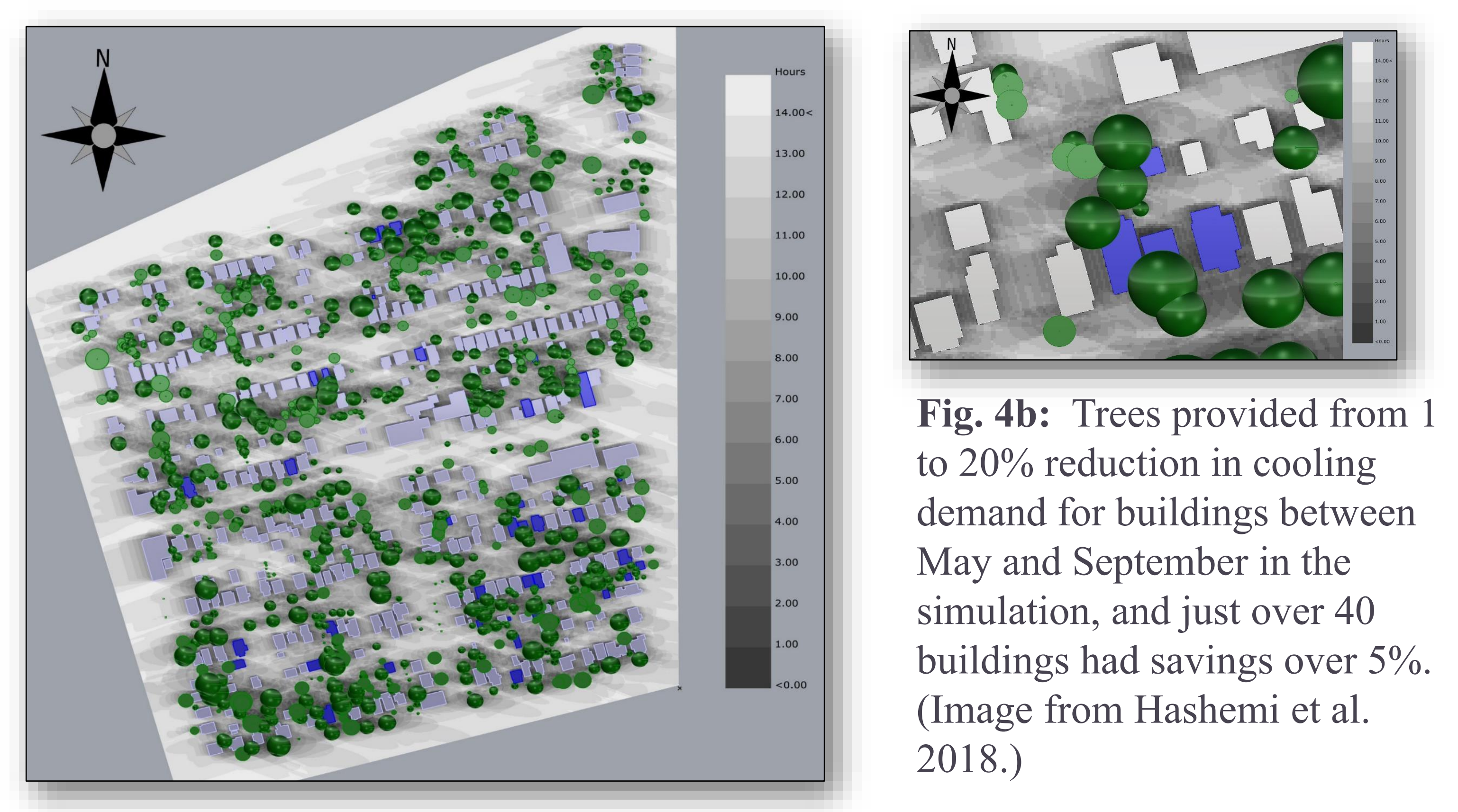

Fig. 4a: Shadow range analysis showing hours of direct sunlight received by buildings; buildings shown in blue are those with $>5 \%$ reduction in cooling demand due to presence of trees. Analyses completed with using Rinoceros input to the umi simulator. (l

\section{$\sum$ References} 1. Allen, R., 1998. FAO Irrigation and Drainage Paper 56. Food and
Agriculture Organization of the United Nations.

4. Hashemi, F., B. Marmur, U. Passe, \& J. Thompson. 2018. Proc.
Simulation for Architecture and Urban Design pp. 331-336. 4. 5imulation, B. Wrike

Food and Agriculture Organization (FAO-UN), n.d. FAO ET Calculator. 6. Available at: http://www.fao.org/land-water/databases-and-software//to-
(1): 2 .

Melillo, J., T. Richmond, \& G. Yohe (eds.). 2014. U.S. Global Change Research Program. 841 pp. doi:10.7930/J0Z31 WJ2. environment

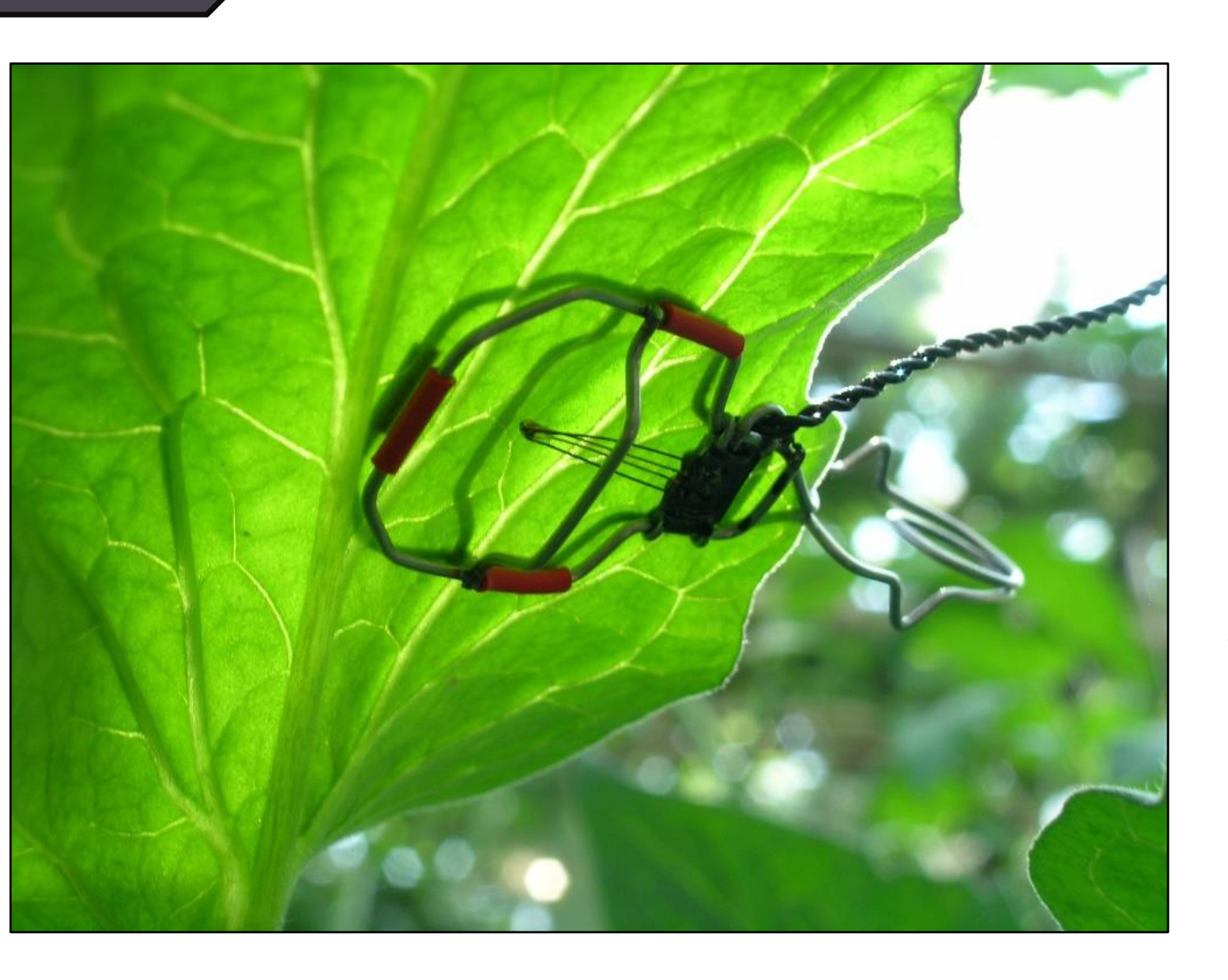
Hg. 3: Leaf temperature probe used to measure absolute emperature of a leaf. Note: This study relied on previously
published leaf temperatures. (Image from www.envoglobal.com.)

In hot and humid climates leaf surface temperatures (Fig. 3) are generally above ambient temperatures due to absorbed solar radiation (Ansari 1959; Vogel 2009). Based on published literature and typical weather conditions in Des Moines, leaf temperatures for tree species in the Capitol East neighbourhood were estimated to be $10^{\circ} \mathrm{C}$ above ambient temperature at noon on a typical sunny day with average wind speeds of $5 \mathrm{mph}$

\section{CFD Preliminary Results}

Depending on weather conditions, buoyancy-driven thermal plumes exist due to ET from trees. The accurate modelling of this phenomenon is critical to evaluating temperature effects on the near-building

- Simulations were performed on a high-performance computer system (TACC Stampede) using a unique octree-based Cartesian meshing scheme (Figs. 5a and 5b)

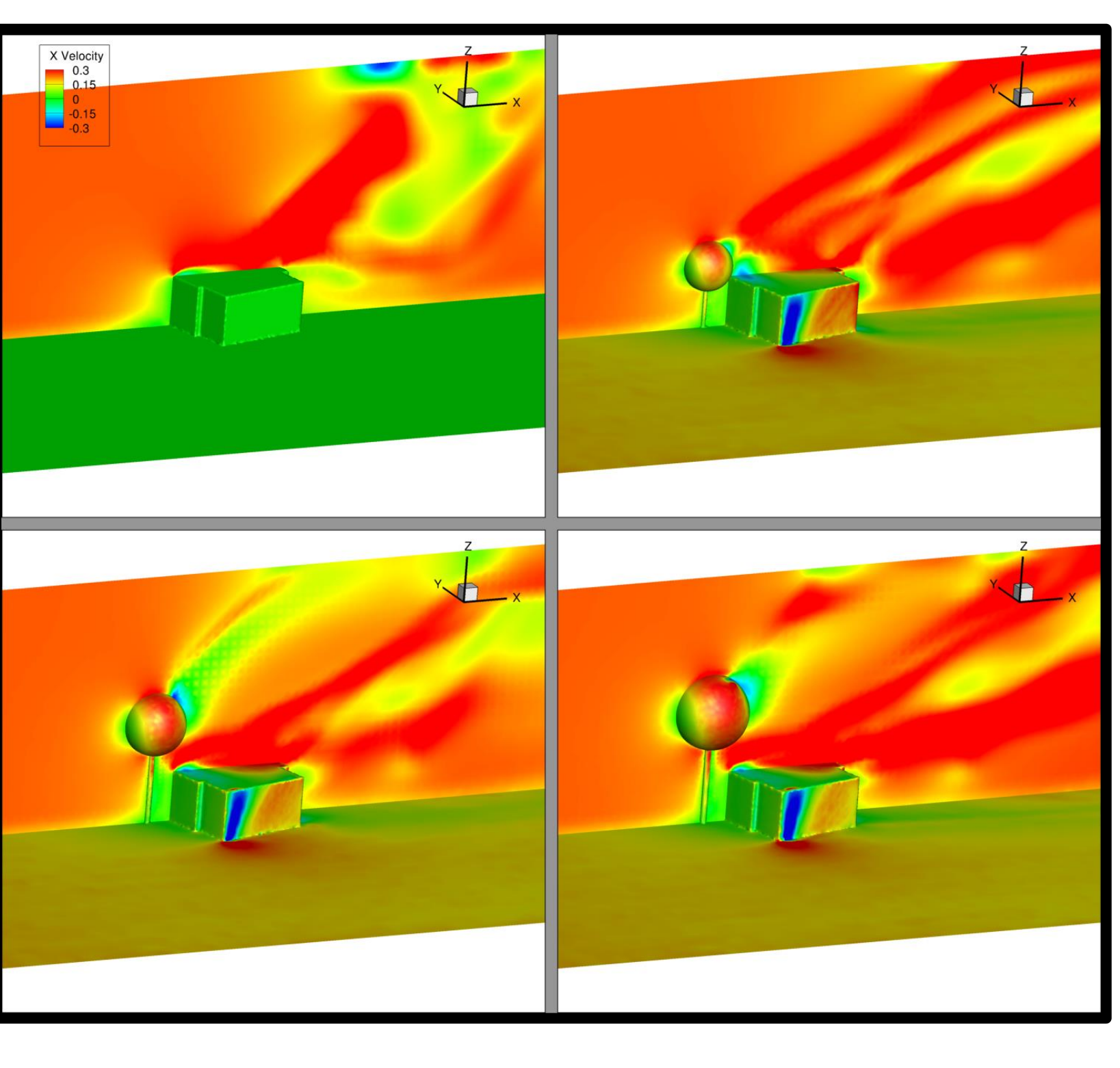

Fig. 5a: Comparison of $\mathrm{x}$-velocity for house only (top left) and three different size trees close to the ho
from Passe et al. 2019.)

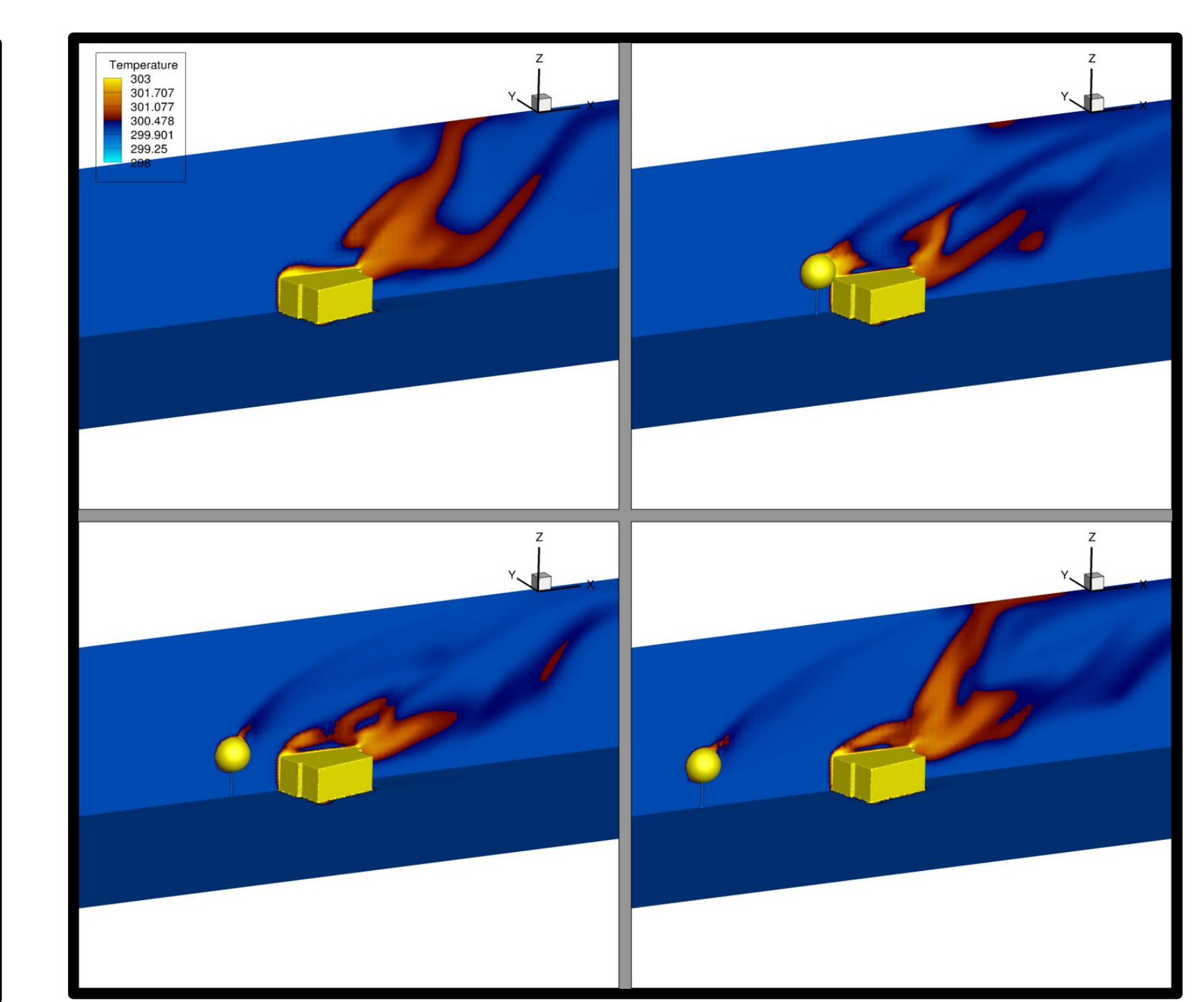

Fig. 5b: Comparison of temperature iso-
surface of house only (top left) and three surface of house only (top left) and three
different size trees close to the house. different size trees close to the house
(Image from Passe et al. 2019.) 